\title{
Efeitos colaterais associados ao tratamento da Leucemia Promielocítica Aguda:
}

\section{revisão sistemática}

\author{
Side effects associated with treatment of Acute Promielocytic Leukemia: systematic review \\ Efectos secundarios asociados al tratamiento de la Leucemia Promielocítica Aguda: una revisión
} sistemática

Recebido: 12/02/2022 | Revisado: 19/02/2022 | Aceito: 21/02/2022 | Publicado: 03/03/2022

\author{
Anderson Silva Salles Filho \\ ORCID: https://orcid.org/0000-0002-7755-8665 \\ Universidade do Estado de Minas Gerais, Brasil \\ E-mail: anderson.1291679@discente.uemg.br \\ Helton Oliveira Campos \\ ORCID: https://orcid.org/0000-0001-5720-8054 \\ Universidade do Estado de Minas Gerais, Brasil \\ E-mail: helton.campos@uemg.br
}

\begin{abstract}
Resumo
A Leucemia Promielocítica Aguda (LPA) é um tipo de câncer que ataca principalmente as células sanguíneas e necessita de tratamentos intensivos que agridem e afetam de forma direta a saúde. Os tratamentos utilizados para controlar a LPA associados aos fármacos, afetam o indivíduo, manifestando efeitos colaterais. Esta revisão sistemática teve por objetivo analisar os efeitos colaterais associados aos tratamentos utilizados para a LPA. Para isto, uma revisão sistemática foi conduzida de acordo com as diretrizes do PRISMA (Preferred Reporting Items for Systematic Reviews and Meta-Analysis). A pesquisa bibliográfica foi realizada nas seguintes bases de dados: PubMed e Web of Science utilizando combinações das seguintes palavras-chave em inglês e português: acute myeloid leukemia, promyelocytic leukemia, M3, treatment, side effects and drugs. Foram incluídos na revisão sistemática estudos que atenderam aos seguintes critérios: (I) os participantes apresentavam diagnóstico de LPA, (II) os pacientes foram submetidos a um protocolo de tratamento para a patologia, (III) os efeitos colaterais associados ao tratamento foram descritos. Um total de 31 artigos foram selecionados para inclusão na revisão sistemática. Os efeitos colaterais mais frequentes associados ao tratamento da LPA encontrados foram: reações cutâneas, desconfortos gastrointestinais, náuseas, vômitos e enxaqueca. A partir dos resultados encontrados nesta revisão sistemática, observou- se que alguns efeitos colaterais aparecem frequentemente nos pacientes com LPA, independentemente do tipo de tratamento utilizado, sendo eles: náuseas, vômitos, reações cutâneas, enxaqueca e desconfortos gastrointestinais.
\end{abstract}

Palavras-chave: Terapêutica; Fármacos hematológicos; Leucemia mieloide aguda.

\begin{abstract}
Acute Promyelocytic Leukemia (APL) is a type of cancer that mainly attacks blood cells and requires intensive treatments that damage and directly affect health. The treatments used to control APL associated with drugs affect the individual, manifesting side effects. This systematic review aimed to analyze the side effects associated with the treatments used for APL. For this, a systematic review was conducted according to the PRISMA guidelines (Preferred Reporting Items for Systematic Reviews and Meta-Analysis). The literature search was carried out in the following databases: PubMed and Web of Science using combinations of the following keywords in English and Portuguese: acute myeloid leukemia, promyelocytic leukemia, M3, treatment, side effects and drugs. Studies that met the following criteria were included in the systematic review: (I) the participants were diagnosed with APL, (II) the patients underwent a treatment protocol for the pathology, (III) the side effects associated with the treatment were described. A total of 31 articles were selected for inclusion in the systematic review. The most frequent side effects associated with the treatment of APL found were: skin reactions, gastrointestinal discomfort, nausea, vomiting and migraine. Based on the results found in this systematic review, it was observed that some side effects frequently appear in patients with APL, regardless of the type of treatment used, namely: nausea, vomiting, skin reactions, migraine and gastrointestinal discomfort.
\end{abstract}

Keywords: Therapeutics; Hematologic agents; Acute myeloid leukemia.

\section{Resumen}

La leucemia promielocítica aguda (LPA) es un tipo de cáncer que ataca principalmente a las células sanguíneas y requiere tratamientos intensivos que atacan y afectan directamente a la salud. Los tratamientos utilizados para el control de la ALI asociados a fármacos afectan al individuo, manifestando efectos secundarios. Esta revisión 
sistemática tuvo como objetivo analizar los efectos secundarios asociados con los tratamientos utilizados para ALI. Para ello, se realizó una revisión sistemática de acuerdo con las guías PRISMA (Preferred Reporting Items for Systematic Reviews and Meta-Analysis). La búsqueda bibliográfica se realizó en las siguientes bases de datos: PubMed y Web of Science utilizando combinaciones de las siguientes palabras clave en inglés y portugués: leucemia mieloide aguda, leucemia promielocítica, M3, tratamiento, efectos secundarios y fármacos. Se incluyeron en la revisión sistemática los estudios que cumplieron con los siguientes criterios: (I) los participantes tenían un diagnóstico de LPA, (II) los pacientes se sometieron a un protocolo de tratamiento para la patología, (III) se describieron los efectos secundarios asociados al tratamiento. Se seleccionaron un total de 31 artículos para su inclusión en la revisión sistemática. Los efectos secundarios más frecuentes asociados al tratamiento de la APL encontrados fueron: reacciones cutáneas, molestias gastrointestinales, náuseas, vómitos y migraña. Con base en los resultados encontrados en esta revisión sistemática, se observó que algunos efectos secundarios aparecen con frecuencia en pacientes con LPA, independientemente del tipo de tratamiento utilizado, a saber: náuseas, vómitos, reacciones cutáneas, migraña y molestias gastrointestinales.

Palabras clave: Terapéutica; Fármacos hematológicos; Leucemia mieloide aguda.

\section{Introdução}

A leucemia é uma patologia resultante da proliferação descontrolada dos glóbulos brancos e se origina na medula óssea, onde as células sanguíneas são substituídas por células leucêmicas patológicas. Estas células leucêmicas preenchem o espaço medular e provocam a inibição do crescimento e da maturação normal dos precursores linfocitários, eritróides, mielóides e megacariocitários. A substituição dos elementos sanguíneos normais por leucócitos leucêmicos leva ao quadro clínico reservado das leucemias, caracterizada por anemia, trombocitopenia e infecções resultantes pela perda de leucócitos funcionantes (Lane, 2010). Esta patologia apresenta quatro variações que são consideradas primárias, sendo elas: Leucemia Mielóide Aguda (LMA), Leucemia Mielóide Crônica (LMC), Leucemia Linfocítica Aguda (LLA) e Leucemia Linfocítica Crônica (LLC) (Nascimento, et al., 2016).

Dentre estas, a Leucemia Mielóide Aguda (LMA) é uma doença hematológica clonal agressiva, que apresenta um bloqueio na diferenciação das células progenitoras hematopoiéticas e alteração da regulação da proliferação celular. Como consequência da produção anormal de células da medula óssea ocorre insuficiência hematopoiética (granulocitopenia, trombocitopenia ou anemia), com ou sem leucocitose (Fröhling, et al., 2005). Baseando-se na disposição celular e nas características citoquímicas dos blastos a LMA pode ser classificada nos seguintes subtipos: leucemia indiferenciada (M0), leucemia mielóide aguda sem diferenciação (M1), leucemia mielóide aguda com diferenciação (M2), leucemia promielocítica aguda (M3), leucemia mielomonocítica (M4), leucemia monocítica (M5), leucemia monocítica sem diferenciação (M5a), leucemia monocítica com diferenciação (M5b), eritroleucemia (M6) e leucemia megacariocítica (M7) (Bennett, et al., 1985).

Os tratamentos farmacológicos da LMA são realizados a partir da combinação de citarabina e daunorrubicina, que apesar de sua taxa de remissão inicial ser notável (cerca de 70 a 85\%), ao longo prazo tende a apresentar efeitos colaterais que comprometem a sobrevida de seus pacientes, devido a toxicidade dessas drogas (Karathedath, et al., 2017). Dentre os diversos subtipos da LMA somente a Leucemia Promielocítica Aguda (LPA - M3) apresenta variação na forma de tratamento (De Kouchkovsky \& Abdul-Hay, 2016) envolvendo não somente a quimioterapia e a drogas tradicionais como as antraciclinas, mas também a aplicação via oral de ácido trans-retinóico (ATRA). Com o auxílio do ATRA o processo de diferenciação granulocítica é retomado, combatendo o efeito da translocação PML-RARa. Por esse motivo a LPA é altamente tratável e de bom prognóstico, tendo um tratamento eficiente na remissão tanto primária quanto secundária da doença (Almeida, 2015).

Desta forma, torna-se interessante analisar de forma individualizada a LPA. Esta variação ocorre devido a alterações estruturais no cromossomo 17, envolvendo o receptor alfa do ácido retinóico (RARa) e o cromossomo 15, que contém o gene Promyelocytic Leukemia (PML) (Leal, et al., 2009). A função do gene PML é atuar como supressor de tumor, impedindo que as células cresçam e multipliquem rapidamente de maneira desordenada. A partir do momento em que há problemas com a 
fusão PML-RARa, as funções originais são perdidas, ocorrendo uma intensa multiplicação das células sem diferenciação e resultando em um tumor (Almeida, 2015).

Mediante a submissão dos pacientes aos medicamentos e aos processos de indução e remissão, alguns efeitos colaterais podem ser apresentados, sendo os mais comuns: náuseas, vômitos, mielossupressão, dermatite, diarreia e mucosite oral (Rubira \& Santos, 2019). Esses efeitos interferem diretamente na qualidade de vida desses pacientes, além de possibilitar o aparecimento de algumas infecções que podem vir a ser fatais devido ao estado debilitado no qual se encontram (Nascimento, et al., 2016). Desta forma, este trabalho tem por objetivo revisar de forma sistemática os efeitos colaterais associados aos tratamentos utilizados para a Leucemia Promielocítica Aguda.

\section{Metodologia}

\subsection{Estratégia de pesquisa}

Neste estudo, foi realizada uma revisão sistemática a partir da pesquisa bibliográfica nas seguintes bases de dados: PubMed e Web of Science, utilizando combinações das seguintes palavras-chave em inglês e português: acute myeloid leukemia, promyelocytic leukemia, M3, treatment, side effects and drugs. Adicionalmente foi realizada a busca manual nas referências dos estudos incluídos na pesquisa. A pesquisa bibliográfica foi realizada entre os anos de 2000 a março de 2021 e não houve restrição quanto ao idioma. A revisão sistemática foi conduzida de acordo com as diretrizes do PRISMA (Preferred Reporting Items for Systematic Reviews and Meta-Analysis) (Matthew, et al., 2021).

\subsection{Seleção dos estudos}

A seleção dos estudos foi realizada por 2 pesquisadores independentes, seguindo as seguintes etapas: exclusão de duplicatas, leitura de títulos, leitura dos resumos e leitura dos artigos na íntegra. Foram incluídos na revisão sistemática estudos que atenderam aos seguintes critérios: (I) os participantes apresentavam diagnóstico de Leucemia Promielocítica aguda, (II) os pacientes foram submetidos a um protocolo de tratamento para a patologia, (III) os efeitos colaterais associados ao tratamento foram descritos. Estudos de revisão e opiniões de especialistas não foram incluídos. Baseando-se nestes critérios de inclusão/exclusão 31 artigos foram selecionados para inclusão nesta revisão sistemática (Figura 1).

\subsection{Extração dos dados}

Foram extraídos dos artigos as seguintes informações: autor e ano do artigo, diagnóstico dos pacientes, número amostral (n), idade, medicamentos utilizados e efeitos colaterais. Estas informações foram organizadas em uma tabela para caracterizar os estudos incluídos. 
Figura 1. Fluxograma do processo de seleção dos estudos, de acordo com o PRISMA.

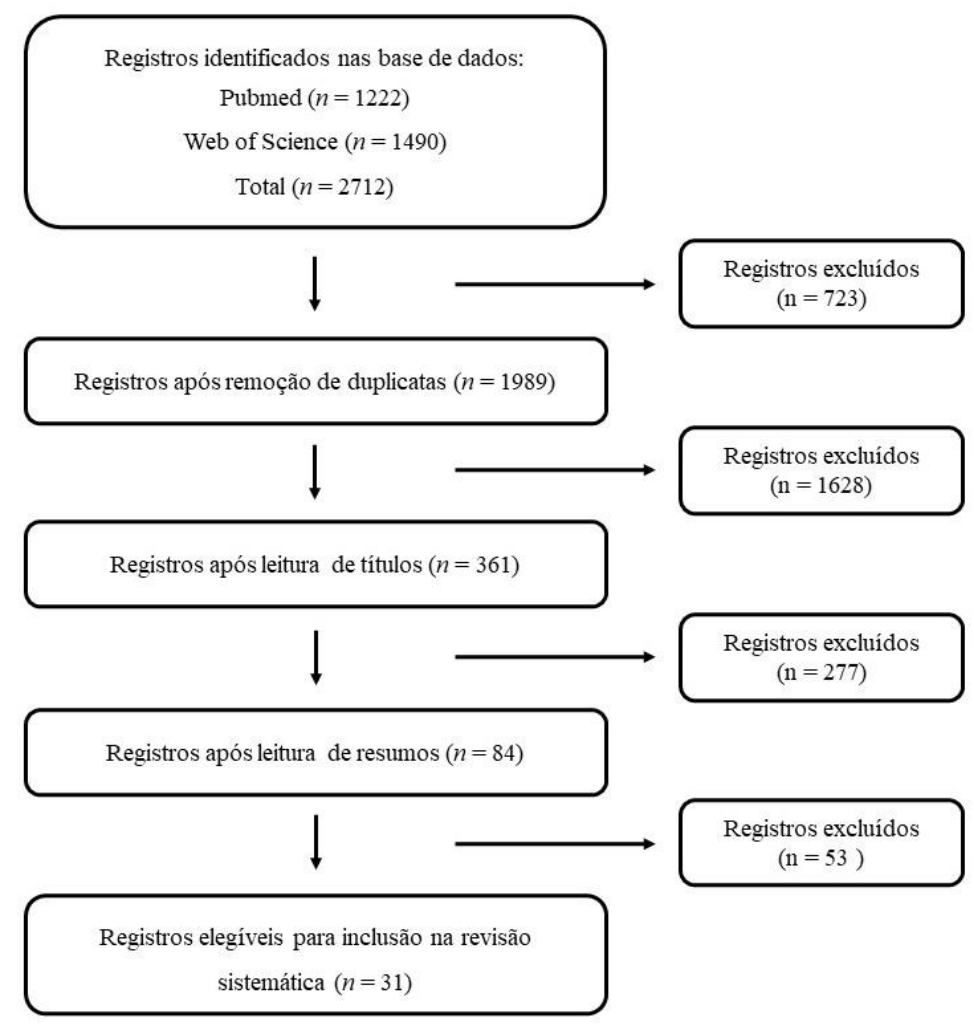

Fonte: Autores

\section{Resultados}

Um total de 2712 artigos foram identificados na pesquisa bibliográfica nas bases de dados. O processo de seleção foi realizado em quatro etapas, utilizando critérios pré-definidos, sendo elas: exclusão de duplicatas (723 artigos excluídos), leitura dos títulos (1628 artigos excluídos), leitura dos resumos (277 artigos excluídos) e leitura do artigo na íntegra (53 artigos excluídos) (Figura 1). Ao final do processo foram selecionados 31 artigos que são apresentados e caracterizados na Tabela 1.

Uma amostra total de 1888 pacientes com Leucemia Promielocítica Aguda foi analisada nos 31 estudos. A população apresentou ampla variação da idade (1 - 82 anos). Apesar da constante evolução sobre os estudos envolvendo a doença, a base do tratamento em sua maioria é realizada a partir dos mesmos fármacos, sendo eles: O ácido trans-retinóico (ATRA) (71,9 \% dos estudos), idarrubicina (18,8\% dos estudos) e o trióxido de arsênio (28,1\% dos estudos), aliados com a quimioterapia $(18,8 \%$ dos estudos).

Dentre os efeitos colaterais mais frequentes associados ao tratamento da LMA foram encontrados os seguintes: reações cutâneas (40,6\% dos estudos), desconfortos gastrointestinais (28,1\% dos estudos), náuseas (12,5\% dos estudos), vômitos $(12,5 \%$ dos estudos) e enxaqueca (12,5\% dos estudos). Outros efeitos colaterais descritos foram a hemorragia, inclusive na área cerebral e doenças envolvendo o bom funcionamento do coração, podendo ser causa de insuficiência cardíaca no paciente. 
Research, Society and Development, v. 11, n. 3, e45711326914, 2022

(CC BY 4.0) | ISSN 2525-3409 | DOI: http://dx.doi.org/10.33448/rsd-v11i3.26914

Tabela 1 - Caracterização dos estudos incluídos na revisão sistemática.

\begin{tabular}{|c|c|c|c|c|c|}
\hline Referência & Diagnóstico dos pacientes & $\mathrm{n}$ & Idade & Medicamentos utilizados & Efeitos colaterais \\
\hline Aldapt, et al., 2018 & LPA & 1 & 23 & ATRA & $\begin{array}{l}\text { - Equimoses nos braços, pernas eabdômen } \\
\text { - Epistases ocasionais } \\
\text { - Sangramentos gengivais }\end{array}$ \\
\hline Beaumont, et al., 2015 & LPA & 106 & $55(12-82)$ & $\begin{array}{c}\text { Quimioterapia } \\
\text { ATRA } \\
\text { Daunorubicina } \\
\text { Citarabina } \\
\text { Mitoxantrona } \\
\text { Ifosfamida } \\
\end{array}$ & $\begin{array}{l}\text { - Insuficiência cardíaca } \\
\text { - Sepse } \\
\text { - Tumores primários }\end{array}$ \\
\hline Buligon, et al., 2018 & LPA & 1 & 32 & ATRA & - Úlceras genitais e orais \\
\hline Candoni, et al., 2018 & LPA & 36 & $37(17-72)$ & $\begin{array}{c}\text { Daunorubicina } \\
\text { ATRA } \\
\text { Citararbina } \\
\text { Idarrubicina } \\
\text { Etoposido } \\
\text { Mitoxantrona }\end{array}$ & $\begin{array}{l}\text { - Febre } \\
\text { - Dificuldade respiratória } \\
\text { - Derrame pleural ou pericárdico } \\
\text { - Infiltração pulmonar intersticial } \\
\text { - Ganho de peso }\end{array}$ \\
\hline Charles, et al., 2000 & LPA & 2 & $34(20-48)$ & $\begin{array}{c}\text { Quimioterapia } \\
\text { ATRA }\end{array}$ & - Úlceras escrotais \\
\hline Chim, et al., 2000 & LPA & 276 & 41 & Quimioterapia & $\begin{array}{l}\text { - Doença isquêmica do coração } \\
\text { - Insuficiência cardíaca congestiva }\end{array}$ \\
\hline Choi, et al., 2011 & LPA & 1 & 39 & $\begin{array}{c}\text { Antraciclina } \\
\text { Quimioterapia } \\
\text { ATRA }\end{array}$ & - Edema pretibial leve \\
\hline Dai, et al., 2009 (1) & LPA & 72 & $34(14-69)$ & ATRA & $\begin{array}{l}\text { - Hepatotoxicidade } \\
\text { - Erupção cutânea } \\
\text { - Enxaqueca } \\
\text { - Ressecamento dos lábios } \\
\text { - Retenção de líquido } \\
\text { - Síndrome de diferenciação }\end{array}$ \\
\hline Dai, et al., 2009 (2) & LPA & 90 & $32(14-67)$ & $\begin{array}{c}\text { ATRA } \\
\text { Trióxido de arsênio }\end{array}$ & $\begin{array}{l}\text { - Hepatotoxicidade } \\
\text { - Erupção cutânea } \\
\text { - Enxaqueca }\end{array}$ \\
\hline
\end{tabular}


Research, Society and Development, v. 11, n. 3, e45711326914, 2022

(CC BY 4.0) | ISSN 2525-3409 | DOI: http://dx.doi.org/10.33448/rsd-v11i3.26914

\begin{tabular}{|c|c|c|c|c|c|}
\hline & & & & & $\begin{array}{l}\text { - Queilite } \\
\text { - Retenção de líquido }\end{array}$ \\
\hline Dasanu, et al., 2013 & LPA & 1 & 73 & $\begin{array}{c}\text { ATRA } \\
\text { Idarrubicina }\end{array}$ & $\begin{array}{l}\text { - Ressecamento cutâneo } \\
\text { - Leuconiquia transversal }\end{array}$ \\
\hline Di Bona, et al., 2000 & LPA & 622 & $39(1-74)$ & ATRA & $\begin{array}{l}\text { - Hemorragia cerebral } \\
\text { - Hemorragia em partes diversas do } \\
\text { corpo; } \\
\text { - Infecção } \\
\text { - Trombose }\end{array}$ \\
\hline Douer, et al., 2001 & LPA & 69 & $44(5-82)$ & ATRA & $\begin{array}{l}\text { - Enxaqueca } \\
\text { - Ressecamento cutâneo } \\
\text { - Febre } \\
\text { - Náusea } \\
\text { - Estomatite } \\
\text { - Vômito } \\
\text { - Queilite } \\
\text { - Dermatite esfoliativa } \\
\text { - Erupção cutânea } \\
\text { - Mialgia } \\
\text { - Anormalidade nas enzimas } \\
\text { hepáticas } \\
\text { - Dor óssea } \\
\text { - Artralgia } \\
\text { - Pseudotumor cerebral } \\
\text { - LDL elevado } \\
\text { - Hipercolesterolemia } \\
\text { - Diarreia } \\
\text { - Prurido } \\
\text { - Calafrio }\end{array}$ \\
\hline Estey, et al., 2001 & LPA & 34 & - & $\begin{array}{c}\text { ATRA } \\
\text { Idarrubicina }\end{array}$ & $\begin{array}{l}\text { - Enxaqueca } \\
\text { - Ressecamento cutâneo } \\
\text { - Artralgia } \\
\text { - Arritmia cardíaca }\end{array}$ \\
\hline Gallipoli \& Drummond, 2009 & LPA & 1 & 31 & $\begin{array}{c}\text { ATRA } \\
\text { Idarrubicina }\end{array}$ & - Pseudotumor cerebral \\
\hline Labrador, et al., 2012 & LPA & 1 & 15 & ATRA & - Papiledema bilateral \\
\hline
\end{tabular}


Research, Society and Development, v. 11, n. 3, e45711326914, 2022

(CC BY 4.0) | ISSN 2525-3409 | DOI: http://dx.doi.org/10.33448/rsd-v11i3.26914

\begin{tabular}{|c|c|c|c|c|c|}
\hline & & & & Idarrubicina & $\begin{array}{l}\text { - Hipertensão intracraniana } \\
\text { - Neuropatia cranial }\end{array}$ \\
\hline Lazo, et al., 2003 & LPA & 12 & - & Trióxido de arsênio & $\begin{array}{l}\text { - Dor de cabeça } \\
\text { - Fadiga } \\
\text { - Erupção cutânea } \\
\text { - Dor torácica } \\
\text { - Dor epigástrica } \\
\text { - Neuropatia }\end{array}$ \\
\hline Leblebjian, et al., 2013 & LPA & 60 & $44(18-79)$ & ATRA & - Pseudotumor cerebral \\
\hline Lengfelder, et al., 2000 & LPA & 51 & $43(16-60)$ & $\begin{array}{l}\text { Quimioterapia } \\
\text { ATRA }\end{array}$ & $\begin{array}{l}\text { - Infecções } \\
\text { - Hemorragia } \\
\text { - Nauseas } \\
\text { - Vômitos } \\
\text { - Diarreia } \\
\text { - Hepatotoxicidade } \\
\text { - Nefrotoxicidade }\end{array}$ \\
\hline Lin, et al., 2000 & LPA & 1 & 37 & ATRA & - Edema de membros inferiores \\
\hline Liu, et al., 2015 & LPA & 31 & $38(17-55)$ & $\begin{array}{c}\text { Quimioterapia } \\
\text { Realgar Natural Indigo Tablets }\end{array}$ & $\begin{array}{l}\text { - Hemorragia cutânea, nasal e oral } \\
\text { - Hiperleucocitose } \\
\text { - Nauseas } \\
\text { - Vômito } \\
\text { - Dor de cabeça } \\
\text { - Ressecamento cutâneo } \\
\text { - Prurido } \\
\text { - Alterações eritomatosas } \\
\text { - Ulceras orais } \\
\text { - Dor de dente }\end{array}$ \\
\hline Lu, et al., 2002 & LPA & 129 & - & Tetra-sulfeto de tetra-arsênio & $\begin{array}{l}\text { - Prolongamento do intervalo QT } \\
\text { - Elevação do nível das enzimas } \\
\text { hepáticas } \\
\text { - Desconforto gastrointestinal } \\
\text { - Erupção cutânea } \\
\text { - Derrame pericárdico }\end{array}$ \\
\hline Machner, et al., 2008 & LPA & 1 & 20 & ATRA & $\begin{array}{l}\text { - Hipertensão intracraniana } \\
\text { - Pseudotumor cerebral }\end{array}$ \\
\hline
\end{tabular}


Research, Society and Development, v. 11, n. 3, e45711326914, 2022

(CC BY 4.0) | ISSN 2525-3409 | DOI: http://dx.doi.org/10.33448/rsd-v11i3.26914

\begin{tabular}{|c|c|c|c|c|c|}
\hline & & & & & - Papiledema bilateral \\
\hline Onishi, et al., 2002 & LPA & 14 & $48(23-65)$ & Trióxido de arsênio & $\begin{array}{l}\text { - Reações gastrointestinais } \\
\text { - Anormalidades eletrocardiográficas } \\
\text { - Reação cutânea } \\
\text { - Neuropatia periférica } \\
\text { - Retenção de fluídos }\end{array}$ \\
\hline Park, et al., 2001 & LPA & 1 & 35 & ATRA & $\begin{array}{l}\text { - Síndrome de Sweet (febre e lesões cutâneas } \\
\text { eritematosas) }\end{array}$ \\
\hline Pavithran, et al., 2004 & LPA & 1 & 13 & ATRA & - Ulceração escrotal \\
\hline Paydas, et al., 2003 & LPA & 1 & 26 & $\begin{array}{c}\text { ATRA } \\
\text { Idarrubicina }\end{array}$ & - Vasculite \\
\hline Shen, et al., 2004 & LPA & 61 & $34(14-74)$ & $\begin{array}{c}\text { ATRA } \\
\text { Trióxido de arsênio }\end{array}$ & $\begin{array}{l}\text { - Reação cutânea } \\
\text { - Reação gastrointestinal } \\
\text { - Boca seca } \\
\text { - Dor de cabeça } \\
\text { - Hiperleucocitose } \\
\text { - Disfunção hepática }\end{array}$ \\
\hline Soignet, et al., 2001 & LPA & 40 & - & Trióxido de arsênio & $\begin{array}{l}\text { - Hipocalemia } \\
\text { - Hiperglicemia } \\
\text { - Neutropenia } \\
\text { - Náusea } \\
\text { - Tosse } \\
\text { - Fadiga } \\
\text { - Enxaqueca } \\
\text { - Vômitos } \\
\text { - Taquicardia } \\
\text { - Diarreia } \\
\text {-Erupção cutânea }\end{array}$ \\
\hline Tao, et al., 2018 & LPA & 70 & $44(12-74)$ & $\begin{array}{c}\text { ATRA } \\
\text { Trióxido de arsênio }\end{array}$ & $\begin{array}{l}\text { - Infecções } \\
\text { - Hemorragia }\end{array}$ \\
\hline Wang, et al., 2011 & LPA & 23 & $40(16-62)$ & Trióxido de arsênio & $\begin{array}{l}\text { - Reação cutânea } \\
\text { - Reação gastrointestinal } \\
\text { - Edema Facial }\end{array}$ \\
\hline Yang, et al., 2018 & LPA & 1 & 22 & Quimioterapia & - Neovascularização macular coroidal \\
\hline
\end{tabular}


Research, Society and Development, v. 11, n. 3, e45711326914, 2022

(CC BY 4.0) | ISSN 2525-3409 | DOI: http://dx.doi.org/10.33448/rsd-v11i3.26914

\begin{tabular}{|c|c|c|c|c|c|}
\hline & & & & $\begin{array}{c}\text { Tretioninina } \\
\text { Trióxido de arsênio } \\
\text { Daunorubicina } \\
\text { Citarabina } \\
\end{array}$ & unilateral \\
\hline Zhu, et al., 2001 & LPA & 78 & - & Trióxido de arsênio & $\begin{array}{l}\text { - Erupção cutânea } \\
\text { - Desconforto gastrointestinal } \\
\text { - Disfunção cardíaca } \\
\text { - Neuropatia e edema facial } \\
\text { - Lesão hepática }\end{array}$ \\
\hline
\end{tabular}

Fonte: Autores. 


\section{Discussão}

A presente revisão sistemática teve por objetivo investigar os efeitos colaterais associados aos tratamentos utilizados para a LPA. Baseando-se nos resultados os efeitos colaterais mais frequentes associados ao tratamento da LPA encontrados foram: reações cutâneas (úlceras orais, genitais e ressecamento), desconfortos gastrointestinais (dores estomacais e diarreia), náuseas, vômitos e enxaqueca. Desta forma, foram apresentados os diversos efeitos colaterais associados ao tratamento da LPA e descritos na literatura.

O tratamento tradicional da LPA é composto pela quimioterapia com antraciclinas (Daunorrubicina, Citarabina e Idarrubicina) e aplicação via oral do Ácido trans Retinóico. Em casos de pacientes mais debilitados e com deficiência de componentes sanguíneos importantes como as plaquetas e as células de defesa, há o acréscimo da transfusão de sangue, plaquetas e de medula óssea para minimizar os sintomas da doença e auxiliar na eficácia do tratamento. Apesar dos efeitos colaterais provocados por essa terapêutica serem extensos, a taxa de remissão é considerada notável e satisfatória, apresentando números expressivos nas literaturas publicadas (Almeida, 2015).

As antraciclinas tem um importante papel no auxílio da quimioterapia, desempenhando diversas funções no tratamento da leucemia promielocítica aguda (Sarturi, et al., 2014). Os fármacos que recebem essa denominação são conhecidos por combater o tumor e não permitir um crescimento ainda maior das células cancerígenas, evitando os processos de metástase. Dentro desse grupo há uma subclassificação dos medicamentos, sendo as mais importantes para o tratamento da LPA: citarabina (antimetabólito); daunorrubicina, idarrubicina, doxorrubicina e mitoxantrona (antitumorais). Contudo os pacientes expostos a eles podem apresentar outros tipos de doença causadas pela cardiotoxicidade, como: a fibrose miocárdica ou até infiltração de células cancerígenas no coração, afetando diretamente a saúde e o processo de tratamento, uma vez que se desenvolvido esse problema o indivíduo não será capaz de dar continuidade ao processo, correndo risco de vida (Ortiz, et al., 2017).

A terapêutica tradicional básico envolvendo a quimioterapia aliada as antraciclinas e ao ATRA funciona de maneira incontestável, alcançando taxas de remissão completa de $90 \%$ dos pacientes, sendo que $70 \%$ dos pacientes necessitam somente da primeira linha de tratamento. Apesar do sucesso em promover a remissão da doença de forma rápida, esse tratamento provoca diversos tipos de efeitos colaterais que afetam a saúde do indivíduo que já se encontra debilitado. Os efeitos mais comuns são: náuseas, vômitos, diarreia e hemorragia na pele, gengivas e nariz (Lengfelder, et al., 2000). Além dos mais comuns os pacientes podem apresentar infecções severas, hemorragias cerebrais e trombose, tendo grandes chances de levar a óbito os indivíduos mais afetados negativamente por esse processo de tratamento (Di Bona, et al., 2000).

Alguns métodos de tratamento alternativos se apresentaram eficientes para estes pacientes. O Trióxido de Arsênio (As2O3) é um exemplo, servindo como complemento ou substituto para o ATRA, realizando a mesma função de combater a anomalia na fusão do gene PML-RARa, alcançando uma taxa de remissão completa de $78 \%$ em cerca de 60 dias após o início do procedimento (Ohnishi, et al., 2002). Os pacientes que foram submetidos a essa terapêutica relataram que houveram reações negativas leves, como: náuseas, vômitos, reações cutâneas, dentre elas úlceras escrotais, enxaqueca e reações gastrointestinais. Alguns outros efeitos mais prejudiciais, como as anormalidades eletrocardiográficas e arritmia cardíaca, requerem um monitoramento médico maior, controlando-os e evitando os danos a sobrevida do indivíduo (Choi, et al., 2011; Dasanu, et al., 2013; Mayorga, et al., 2002).

Os comprimidos naturais, também conhecidos como "Compound Realgar Natural Indigo Tablets" (CRNIT), compostos por Savia miltiorrhiza e Radix pseudostellaria, são utilizados como terapia em conjunto com a quimioterapia para tratar a LPA. Esse tipo de terapêutica também se mostrou eficiente, tendo uma taxa de remissão completa de 90,32\%, variando entre 10 a 60 meses após o início do procedimento. Houve manifestação de efeitos colaterais, sendo os mais comuns: náuseas, vômitos, hemorragia na pele, gengivas e nariz, úlcera oral, dor de cabeça, dor de dente e ressecamento da pele. Notou-se 
também a manifestação de hiperleucocitose em cerca de 80 a $90 \%$ dos indivíduos, precisando serem monitorados para não ocorrer o agravamento da doença. (Lu, et al., 2002) Apesar da funcionalidade dessa metodologia, o tempo de resposta para alcançar a taxa de remissão é muito maior do que as apresentadas nesse trabalho (Liu, et al., 2015).

Levando em consideração os fatos acima citados, os tratamentos alternativos vêm sendo estudados e aplicados em diversos países diferentes, demostrando tanto efeitos negativos quanto positivos de seu uso. De maneira geral todos obtiveram resultados notáveis na remissão completa da LPA, uns afetando mais a sobrevida do que outros. O Trióxido de Arsênio aparece cada vez mais como uma alternativa ao ATRA, podendo em alguns casos serem usados em conjunto. Os CRNITs são frutos de estudos mais recentes, mas tem potencial para se tornarem um método terapêutico menos agressivo se melhores estudados, em contrapartida com a resposta mais lenta para chegar à remissão completa da doença. De fato, cada método terapêutico possui suas peculiaridades, influenciando diretamente nos efeitos colaterais manifestados pelos pacientes, sendo necessário um balanço para saber qual tratamento seria melhor para cada caso correlacionando benefícios e malefícios, a fim de garantir uma sobrevida maior aos pacientes de forma segura (Choi, et al., 2011; Liu, et al., 2015).

\section{Conclusão}

A partir dos resultados encontrados nesta revisão sistemática, observou- se que alguns efeitos colaterais aparecem frequentemente nos pacientes com LPA, independentemente do tipo de tratamento utilizado, sendo eles: náuseas, vômitos, reações cutâneas, enxaqueca e desconfortos gastrointestinais. Estes efeitos colaterais apresentam baixo risco para os pacientes, sendo facilmente minimizados. Em contrapartida, efeitos colaterais mais fortes podem estar presentes, sendo eles: cardiotoxicidade, hiperleucocitose e hemorragia cerebral, exigindo um acompanhamento para que não haja complicações e que possam dar continuidade ao processo, uma vez que caso expressem essas doenças o tratamento é interrompido, acarretando risco de vida ao paciente. Estudos visando a descoberta de novos tratamentos associados a menor frequência de efeitos colaterais devem continuar buscando novos fármacos, a fim de oferecer uma melhor qualidade de vida aos portadores da LPA, com o menor número de efeitos colaterais associados.

\section{Referências}

Aldapt, M. B., Kassem, N., Al-Okka, R., Ghasoub, R., Soliman, D., Abdulla, M. A., Mudawi, D., Ibrahim, F. \& Yassin, M. A. (2018). Thrombocytosis in a male patient with acute promyelocytic leukaemia during all-trans retinoic (ATRA) acid treatment. Acta Biomed, 89 (3), 33-7.

Almeida, S. C. Leucemia promielocítica aguda: clínica diagnóstico e abordagem [tese]. Universidade de Coimbra; 2015.

Beaumont, M., Sanz, M., Carli, P. M., Maloisel, F., Thomas, X., Detourmignies, L., Guerci, A., Gratecos, N., Rayon, C., Miguel, J. S., Odriozola, J., Cahn, J. Y., Huguet, F., Vekhof, A., Stamatoulas, A., Dombret, H., Capote, F., Esteve, J., Stoppa, A. M. \& Fenaux, P. (2015). Therapy-related acute promyelocytic leukemia. Journal of Clinical Oncology, 21 (11), 2123-37.

Bennett, J. M., Catovsky, D., Daniel, M. T., Flandrin, G., Galton, D. A., Gralnick, H. R. \& Sultan, C. (1985). Proposed revised criteria for the classification of acute myeloid leukemia. A report of the French-American-British Cooperative Group. Annals of Internal Medicine,103 (4), 620-5.

Buligon, M. P., Mielke, J. C., Chiesa, J. \& Ferrazo, K. L. (2018). Rare labial ulcer related to the use of all-trans retinoic acid in a patient with acute promyelocytic leukemia. Special Care Dentistry, 38 (4), 234-8.

Candoni, A., Damiani, D., Michelutti, A., Masolini, P., Michieli. M., Michelutti, T., Geromin, A. \& Fanin, R. (2003). Clinical characteristics, prognostic factors and multidrug-resistance related protein expression in 36 adult patients with acute promyelocytic leukemia. European Journal of Haematology, 71 (1), $1-8$.

Charles, K. S., Kanaa, M., Winfield, D. A. \& Reilly, J. T. (2000). Scrotal ulceration during all-trans retinoic (ATRA) therapy for acute promyelocytic leukaemia. Clinical \& Laboatory Haematology, 22 (3), 171-4.

Chim, C. S., Liang, R., Kwong, Y. L., Lie, A. K. W., Todd, D. \& Chan, T. K. (2008). Treatment of de novo acute myeloid leukaemia in Hong Kong: a twenty-year experience (1975 to 1996). Australian and New Zealand Journal of Medicine, 29 (5), 726-30.

Choi, S., Kim, H. S., Jung, C. S., Jung, S. W., Lee, Y. J., Rheu, J. K., Jo, J. R. \& Lee, N. H. (2011). Reversible Symptomatic Myocarditis Induced by All-Trans Retinoic Acid Administration during Induction Treatment of Acute Promyelocytic Leukemia: Rare Cardiac Manifestation as a Retinoic Acid Syndrome. Journal of Cardiovasc Ultrasound, 19 (2), 95-8. 
Dai, C. W., Zhang, G. S., Shen, J. K., Zheng, W. L., Pei, M. F., Xu, Y. X., Cao, Y. X., Yi, Y., Yang, J. J., Peng, H. L., Zhong, H. Y. \& Li, R. J. (2009). Use of all-trans retinoic acid in combination with arsenic trioxide for remission induction in patients with newly diagnosed acute promyelocytic leukemia and for consolidation/maintenance in CR patients. Acta Haematologica, 121 (1), 1-8.

Dasanu, C. A., Ichim, T. E. \& Alexandrescu, D. T. (2013). Muehrcke's lines (Leukonychia striata) due to transretinoic acid therapy for acute promyelocytic leukemia. Journal of Oncology Pharmacy Practice, 19 (4), 377-9.

De Kouchkovsky, I. \& Abdul-Hay, M. (2016). Acute myeloid leukemia: a comprehensive review and 2016 update. Blood Cancer Journal, 6 (7), e441.

Di Bona, E., Avvisati, G., Castaman, G., Luce, V. M., De Sanctis, F., Rodeghiero, F. \& Mandeli, F. (2000). Early Haemorrhagic morbidity and mortality during remission induction whit or whitout all-trans retinoic acid in acute promyelocytic leukemia. British Journal of Haematology, 108 (4), 689-95.

Douer, D., Estey, E., Santillana, S., Bennett, J. M., Lopez-Bernstein, G., Boehm, K. \& Williams, T. (2001). Treatment of newly diagnosed and relapsed acute promyelocytic leukemia with intravenous liposomal all-trans retinoic acid. Blood, 97 (1), 73-80.

Estey, E., Koller, C., Cortes, J., Reed, P., Freireich, E., Giles, F. \& Kantarjian, H. (2001). Treatment of newly-diagnosed acute promyelocytic leukemia with liposomal all-trans retinoic acid. Leukemia \& Lymphoma, 42 (3), 309-16.

Fröhling, S., Scholl, C., Gilliland, D. G. \& Levine, R. L. (2005). Genetics of myeloid malignancies: pathogenetic and clinical implications. Journal of Clinical Oncolgy, 23 (26), 6285-95.

Gallipoli, P. \& Drummond, M. W. (2009) Pseudotumour cerebri as a manageable side effect of prolonged all-trans retinoic acid therapy in an adult patient with acute promyelocytic leukaemia. European Journal of Haematology, 82 (3), 242-3.

Karathedath, S., Rajamani, B. M., Aalam, S. M. M., Abraham, A., Varatarajhan, S., Kishnamurthy, P., Mathews, V., Velayudhan, S. R. \& Balasubramanian, P. (2017). Role of NF-E2 related factor 2 (Nrf2) on chemotherapy resistance in acute myeloid leukemia (AML) and the effect of pharmacological inhibition of Nrf2. PLoS One, 12 (5), e0177227.

Labrador, J., Puig, N., Ortín, A., Gutierrez, N. C. \& González-Díaz, M. (2012). Multiple cranial neuropathy and intracranial hypertension associated with alltrans retinoic acid treatment in a young adult patient with acute promyelocytic leukemia. International Journal of Hematology, 96 (3), 383-5.

Lane, S. W. \& Gilliland, D. G. (2010). Leukemia stem cells. Seminar in Cancer Biology, 20 (2), 71-6.

Lazo, G., Kantarjian, H., Estey, E., Thomas, D., O’Brien, S. \& Cortes, J. (2003). Use of arsenic trioxide (As2O3) in the treatment of patients with acute promyelocytic leukemia: the M. D. Anderson experience. Cancer, 97 (9), 2218-24.

Leal, A. M., Kumeda, C. A. \& Velloso, E. D. R. P. (2009). Características genéticas da leucemia promielocítica aguda de novo. Revista Brasileira de Hematologia e Hemoterapia, 31 (6), 1-9.

Leblebjian, H., DeAngelo, D. J., Skirvin, J. A., Stone, R. M., Wadleigh, M., Werner, M., Neuberg, D. S., Bartel, S. \& McDonnell, A. M. (2013). Predictive factors for all-trans retinoic acid-related differentiation syndrome in patients with acute promyelocytic leukemia. Leukemia Research, 37 (7), 747-51.

Lengfelder, E., Reichert, A., Schoch, C., Haase, D., Haferlach, T., Löffler, H., Staib, P., Heyll, A., Seifarth, W., Saussele, C., Fonatsch, C., Gassmann, W., Ludwig, W. D., Hochhaus, A., Beelen, D., Aul, C., Sauerland, M. C., Heinecke, A., Hehlmann, R., Wörmann, B., Hiddemann, W. \& Büchner, T. (2000). Double induction strategy including high dose cytarabine in combination with all-trans retinoic acid: effects in patients with newly diagnosed acute promyelocytic leukemia. Leukemia, 14 (8), 1362-70.

Lin, C. P., Huang, M. J., Chang, I. Y., Lin, W. Y. \& Sheu, Y. T. (2000). Retinoic acid syndrome induced by arsenic trioxide in treating recurrent all-trans retinoic acid resistant acute promyelocytic leukemia. Leukemia \& Lymphoma, 38 (1-2), 195-8.

Liu, Y., He, P., Cheng, X. \& Zhang, M. (2015). Long-term outcome of 31 cases of refractory acute promyelocytic leukemia treated with compound realgar natural indigo tablets administered alternately with chemotherapy. Oncology Letters, 10 (2), 1184-90.

Lu, D. P., Qiu, J. Y., Jiang, B., Wang, Q., Liu, K. Y., Liu, Y. R. \& Chen, S. S. (2002). Tetra- arsenic tetra-sulfide for the treatment of acute promyelocytic leukemia: a pilot report. Blood, 99 (9), 3136-43.

Machner, B., Neppert, B., Paulsen, M., Hofmann, C., Sander, T. \& Helmchen, C. (2018). Pseudotumor cerebri as a reversible side effect of all-trans retinoic acid treatment in acute promyelocytic leukaemia. European Journal of Neurology, 15 (7), 68-9.

Matthew, J. P., Joanne, E. M., Patrick, M. B., Isabelle, B., Tammy, C. H., Cynthia, D. M., Shamseer, L., Tetzlaff, J. M., Akl, E. A., Brennan, S. E., Chou, R., Glanville, J., Grimshaw, J. M., Hróbjartsson, A., Lalu, M. M., Li, T., Loder. E. W., Mayo-Wilson, E., McDonald, S., McGuinness, L. A., Stewart, L. A., Thomas, J., Tricco, A. C., Welch, V. A., Whiting, P. \& Moher, D. (2021). The PRISMA 2020 statement: an updated guideline for reporting systematic reviews. $B M J, 29,372: \mathrm{n} 71$.

Mayorga, J., Richardson-Hardin, C. \& Dicke, K. A. (2002). Arsenic trioxide as effective therapy for relapsed acute promyelocytic leukemia. Clinical Journal of Oncology Nursing, 6 (6), 341-6.

Nascimento, C. A. D., Cartaxo, C. M. B., Lopes, K. A. M., Buhatsky, M. \& Batista, A. F. (2016). Leucemia mieloide aguda (LMA): as condições psicológicas do paciente adulto. Psicologia em Revista, 22 (2), 1677-1168.

Ohnishi K, Yoshida H, Shigeno K, Nakamura S, Fujisawa S, Naito K., Shinjo, K., Fujita, Y., Matsui, H., Sahara, N., Takeshita, A., Satoh, H., Terada, H. \& Ohno, R. (2002). Arsenic trioxide therapy for relapsed or refractory japanese patients with acute promyelocytic leukemia: need for careful electrocardiogram monitoring. Leukemia, 16 (4), 617-22.

Ortiz, J. V., Assunção, B. M. B. L., Crosbie, M. S. \& De Freitas, F. A. (2017). Cardiotoxicidade por antraciclinas em pacientes com leucemia aguda: uma revisão de literatura. Revista Pleiade, 11 (21), 5-16. 
Research, Society and Development, v. 11, n. 3, e45711326914, 2022

(CC BY 4.0) | ISSN 2525-3409 | DOI: http://dx.doi.org/10.33448/rsd-v11i3.26914

Park, C. J., Bae, Y. D., Choi, J. Y., Heo, P. S., Lee, K. S., Park, Y. S. \& Lee, J. A. (2001). Sweet's syndrome during the treatment of acute promyelocytic leukemia with all- trans retinoic acid. The Korean Journal of Internal Medicine, 16 (3), 218-21.

Pavithran, K., Arjun, R., Aruna, R. \& Thomas, M. (2004). Scrotal ulceration during induction therapy of acute promyelocytic leukemia with ATRA. American Journal of Hematology, 75 (4), 260-1.

Paydas, S., Yavuz, S., Disel, U., Sahin, B., Canbolat, T. \& Tuncer, I. (2003). Vasculitis associated with all trans retinoic acid (ATRA) in a case with acute promyelocytic leukemia. Leukemia \& Limphoma, 44 (3), 547-8.

Rubira, C. M. F. \& Santos, P. S. S. Manejo da mucosite oral quimioinduzida em criança com leucemia Mielóide aguda. In: Congresso Odontológico de Bauru; 2019; Bauru.

Sarturi, B. F., Bittencourt, R. I., Spindler, B. M., Contin, L. C. Z., Soares, T. B., Paiva, M. F., Burin, M. M. \& Daudt, L. E. Avaliação de cardiotoxicidade pós uso de antraciclina em pacientes com leucemia mielóide aguda entre os anos de 2007 a 2013. In: 34 $4^{a}$ Semana Científica do Hospital de Clínicas de Porto Alegre; 2014; Porto Alegre.

Shen ZX, Shi ZZ, Fang J, Gu BW, Li JM, Zhu YM., Shi, J. Y., Zheng, P. Z., Yan, H., Liu, Y. F., Chen, Y., Shen, Y., Wu, W., Tang, W., Waxman, S., Thé, H. D., Wang, Z. Y., Chen, S. J. \& Chen, Z. (2204). All-trans retinoic acid/As2O3 combination yields a high quality remission and survival in newly diagnosed acute promyelocytic leukemia. Proceedings of the National Academy of Sciences, 101 (15), 5328-35.

Soignet SL, Frankel SR, Douer D, Tallman MS, Kantarjian H, Calleja E., Stone, R. M., Kalaycio, M., Scheinberg, D. A., Steinherz, P., Sievers, E. L., Coutré, S., Dahlberg, S., Ellison, R. \& Warrell, R. P. (2001). United states multicenter study of arsenic trioxide in relapsed acute promyelocytic leukemia. Journal of Clinical Oncology, 19 (18), 3852-60.

Tao, S., Wang, C., Chen, Y., Deng, Y., Song, L., Shi, Y., Lin, L., Ding, B., He, Z. \& Yu, L. (2018). Long-term effect of all-trans retinoic acid and arsenic trioxide sequential maintenance in patients with acute promyelocytic leukemia. Leukemia \& Lymphoma, 60 (3), 711-9.

Wang, H., Xi, S., Liu, Z., Yang, Y., Zheng, Q., Wang, F., Xu, Y., Wang, Y., Zheng, Y. \& Sun, G. (2011). Arsenic methylation metabolism and liver injury of acute promyelocytic leukemia patients undergoing arsenic trioxide treatment. Environmental Toxicology, 28 (5), 267-75.

Yang, X., Xu, J., Yang, J., Zhou, Y., Mei, Y., Yang, T. \& Zhang, Y. (2018). Unilateral macular choroidal neovascularization-a rare manifestation in acute myelocytic leukemia. Medicine, 97 (16), e0344.

Zhu, C., Guo-Qiyang, C., Zhi-Xiang, S., Sai-Juan, C. \& Zhen-Yi, W. (2001). Treatment of acute promyelocytic leukemia with arsenic compounds: In vitro and in vivo studies. Seminars in Hematology, 38 (1), 26-36. 